\title{
ARTICLE OPEN \\ Persistence of spin memory in a crystalline, insulating phase-change material
}

\author{
Johannes Reindl ${ }^{1}$, Hanno Volker ${ }^{1}$, Nicholas P. Breznay ${ }^{2}$ and Matthias Wuttig $\mathbb{D}^{1,3 *}$
}

The description of disorder-induced electron localization by Anderson over 60 years ago began a quest for novel phenomena emerging from electronic interactions in the presence of disorder. Even today, the interplay of interactions and disorder remains incompletely understood. This holds in particular for strongly disordered materials where charge transport depends on 'hopping' between localized sites. Here we report an unexpected spin sensitivity of the electrical conductivity at the transition from diffusive to hopping conduction in a material that combines strong spin-orbit coupling and weak inter-electronic interactions. In thin films of the disordered crystalline phase change material $\mathrm{SnSb}_{2} \mathrm{Te}_{4}$, a distinct change in electrical conductance with applied magnetic field is observed at low temperatures. This magnetoconductance changes sign and becomes anisotropic at the disorder-driven crossover from strongly localized (hopping) to weakly localized (diffusive) electron motion. The positive and isotropic magnetoconductance arises from disruption of spin correlations that inhibit hopping transport. This experimental observation of a recently hypothesized 'spin memory' demonstrates the spin plays a previously overlooked role in the disorder-driven transition between weak and strong localization in materials with strong spin-orbit interactions.

npj Quantum Materials (2019)4:57; https://doi.org/10.1038/s41535-019-0196-6

\section{INTRODUCTION}

Ever since Anderson published his seminal paper on disorderinduced electron localization in 1958, the effects of disorder on wave phenomena have intrigued scientists. ${ }^{1}$ Today, the concept of localization continues to find widespread application in fields ranging from many body theory ${ }^{2}$ to tunable cold atom systems $s^{3,4}$ and classical wave phenomena. ${ }^{5,6}$ Surprisingly, however, Anderson localization is difficult to isolate in the context for which it was originally developed: electrons in disordered solids. This difficulty arises from the intertwined effects of disorder and electronic interactions as well as the inherent complexity in controlling and analytically quantifying disorder. Hence, even the character of the insulating electronic ground state in a disordered solid has not been settled. ${ }^{7-9}$ Particularly for quantum materials whose properties are driven by strong spin-orbit coupling, systematically understanding the impact of disorder on spin-dependent electronic transport when approaching a strongly localized, insulating state remains an outstanding challenge, although in recent years also significant progress has been made. ${ }^{10-12}$

In weakly disordered metals, spin lifetimes have long been studied using the unambiguous signature of electronic quantum interference: weak localization. Quantum corrections to diffusive metallic transport, known as weak localization, appear when disorder influences the physical properties of the material under study but does not yet dominate it. ${ }^{13-16}$ This constructive interference of self-intersecting trajectories impedes transport and thus reduces the conductance of the system; in the presence of strong spin-orbit coupling the trajectories destructively interfere and result in weak antilocalization. ${ }^{13-16}$ Both phenomena appear with unambiguous signatures in magnetotransport that can be used as a spectroscopic probe. ${ }^{13}$ In strongly disordered materials, on the contrary, no similar approach to study spindependent transport and dynamics exists. Below we present such an approach, uncovering evidence for spin-sensitive hopping transport in a strongly localized electronic system.

To systemically explore the spin sensitivity of charge transport with strong disorder, we first identify a class of materials where tunable microscopic randomness exists. Crystalline phase change materials (PCM) such as compounds along the pseudo-binary line between $\mathrm{GeTe}$ and $\mathrm{Sb}_{2} \mathrm{Te}_{3}$ provide a model system to explore disorder-induced localization and show a breadth of disorderdriven phenomena. A metal-insulator transition (MIT) in bulk PCMs (like $\mathrm{GeSb}_{2} \mathrm{Te}_{4}$ ) arises through the ordering of the intrinsic vacancies in the metastable rock-salt phase Fig. $1 a^{17-22}$ preceded by hallmark phase-coherent effects on the metallic side of the $\mathrm{MIT}^{21,23}$ driven by strong spin-orbit interactions. To investigate charge transport beyond the breakdown of the diffusive picture, we consider whether spin- or phase-coherence lifetimes still play a prominent role in the insulating state of phase-change materials as the disorder is continuously increased.

\section{RESULTS}

Electrical transport at low temperatures

$\mathrm{SnSb}_{2} \mathrm{Te}_{4}$ (SST) closely resembles prototypical PCMs such as $\mathrm{GeSb}_{2} \mathrm{Te}_{4}$ or $\mathrm{Ge}_{2} \mathrm{Sb}_{2} \mathrm{Te}_{5}$, which are employed in non-volatile rewriteable optical and electronic memories, ${ }^{24,25}$ in terms of atomic arrangement and physical properties. Like these two compounds, it also allows for precise disorder tuning near the MIT upon annealing. We study a series of thin film $\mathrm{SnSb}_{2} \mathrm{Te}_{4}$ devices pictured in Fig. 1 b (also Supplementary Note 1) with varying levels of disorder tuned by post-growth annealing (see Methods) and characterize their electrical transport properties using standard low-temperature techniques. We begin by examining the most metallic sample S9, whose normal state conductance $G$ at all temperatures (Fig. 1c, d) is much larger than a critical conductance $G_{c} \approx G_{0} / \pi^{2}$ (with $G_{0}=\mathrm{e}^{2} / \hbar$ ) expected from scaling theory. ${ }^{14}$ For 

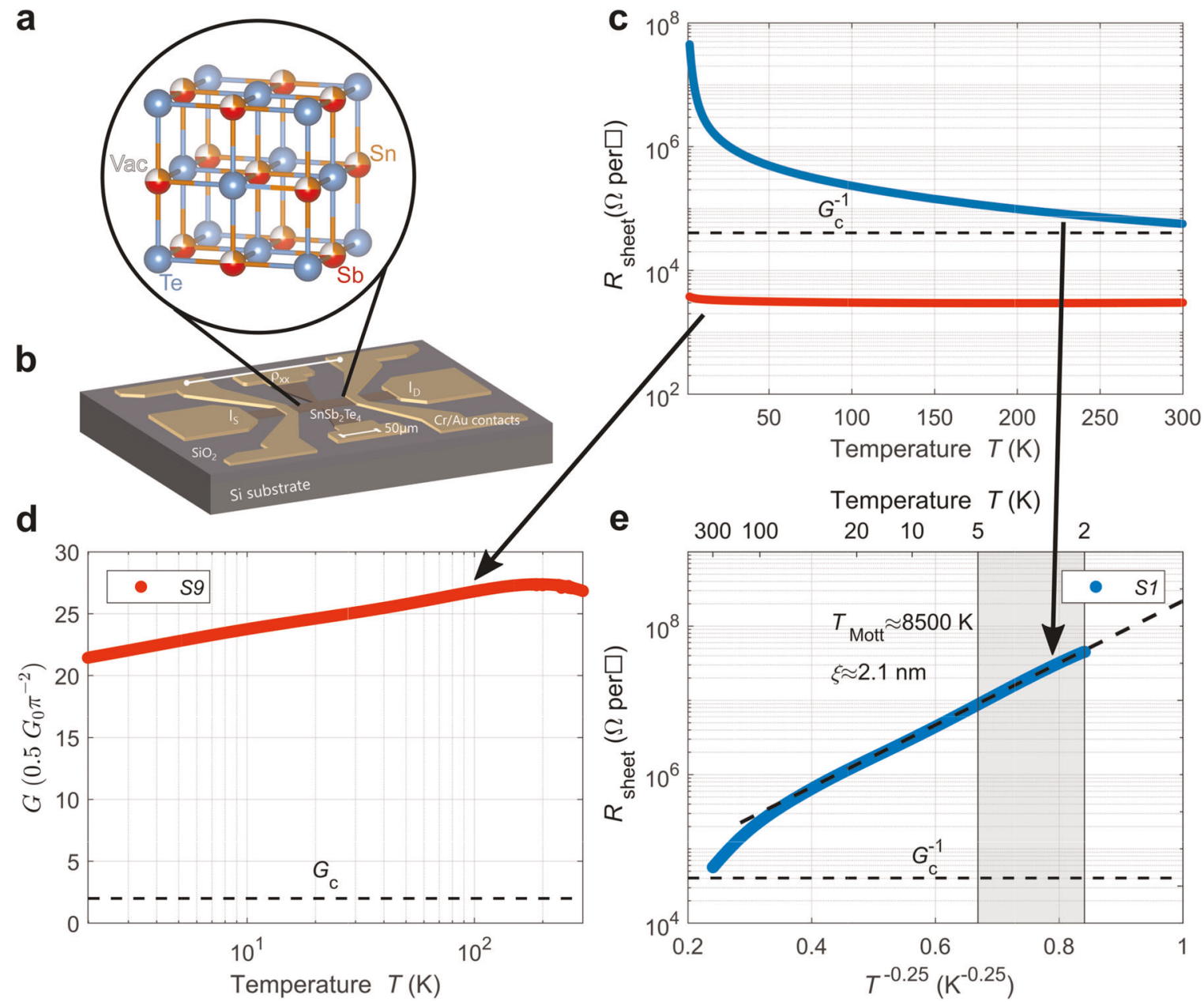

Fig. 1 Temperature dependence of the conductance/ resistance of metallic and insulating samples of $\mathrm{SnSb}_{2} \mathrm{Te}_{4}$. a Crystal structure of (metastable) rock-salt $\mathrm{SnSb}_{2} \mathrm{Te}_{4}$ with Te occupying one sublattice and $25 \%$ vacancies, $25 \% \mathrm{Sn}$ and $50 \% \mathrm{Sb}$ randomly distributed on the other sublattice. The disorder on the formal cation-sublattice causes charge carrier localization. $\mathbf{b}$ Schematic Hall bar device employed for electrical transport experiments; the scale bar is $50 \mu \mathrm{m}$. c Sheet resistance vs. temperature for the most insulating $S 1$ and most conducting $S 9$ samples. d Metallic sample $S 9$ shows a conductance $\left(G>G_{c} \approx G_{0} / \pi^{2}\right)$ decreasing as log $(T)$ for decreasing temperature due to quantum corrections. e Insulating sample $S 1$ shows variable range hopping behavior [In $(R) \sim\left(T / T_{\text {Mott }}\right)^{-1 / 4}$ ] with power-law increase of the resistance below $50 \mathrm{~K}$; $T_{\text {Mott }} \approx 8500 \mathrm{~K}$ for the fitting range marked by the gray background.

three dimensions $G_{c}$ indicates the critical conductance associated with the MIT, not a minimum metallic conductance, whereas for two dimensions a crossover from extended to strongly localized states is expected. Hall effect measurements show a temperatureindependent carrier density of $n=2.3 \times 10^{20} \mathrm{~cm}^{-3}$ (see Supplementary Note 2), and a nearly temperature-independent resistance with a $\log (T)$ contribution shown in Fig. 1c, d indicating that this sample is a diffusive metal with low-temperature conductance corrections. In contrast, the most insulating sample $S 1$ exhibits an exponential temperature dependence characteristic of variable range hopping (VRH) transport (Fig. 1c, e) described by $R \approx R_{0} \exp \left(\left(\frac{T}{T_{M o t t}}\right)^{-\frac{1}{4}}\right)$; the associated carrier localization length of $2.1 \mathrm{~nm}^{8,9}$ also confirms that the sample shows strong-localization. However, the occurrence of a separate Efros-Shklovskii hopping regime indicative of a soft Coulomb gap ${ }^{9}$ and usually present at lowest temperatures ${ }^{19,21,26,27}$ cannot be unambiguously detected (see Supplementary Note 6).

With increasing disorder, the resistance rises continuously in $\mathrm{SnSb}_{2} \mathrm{Te}_{4}$. As shown in Fig. 2, at fixed temperature, rising disorder leads to an increase of sheet resistance $\left(R_{\text {sheet }}\right)$. A transition from diffusive transport to hopping conduction is observed as $R_{\text {sheet }}$ nears $2 \pi^{2} / G_{0}$ (see also Supplementary Note 3 ). To better understand the nature of this transition, we simultaneously track the sign of the magnetoconductance (MC): the color of the markers in Fig. 2 indicates the sign of the low-field $M C$ for perpendicular (upwards triangle) and parallel field (downwards triangle) configurations (see also Supplementary Note 4). The most metallic sample 59 shows a negative MC (green) for both field orientations at all temperatures, while the low-field MC sign for the most insulating sample $S 1$ is positive (blue).

The transition from diffusive to hopping transport is thus accompanied by an unambiguous change in magnetotransport at low temperature. Metallic samples show negative $M C$ arising from weak antilocalization (WAL), while the low-field MC turns positive as the sample resistance increases. At low temperatures, a crossover from diffusive to hopping transport is often accompanied by a clear MC sign change with critical resistance values of $2 \pi / G_{0}{ }^{28,29}$ or $2 \pi^{2} / G_{0}{ }^{30}$ The change of sign is anisotropic with respect to the field direction: negative $M C$ is observed at lower temperatures in perpendicular than in parallel fields. Even more surprisingly, temperatures up to $30 \mathrm{~K}$ show the same $M C$ sign change near $2 \pi^{2} / G_{0}$, raising the question of what process accounts for this evolution. 


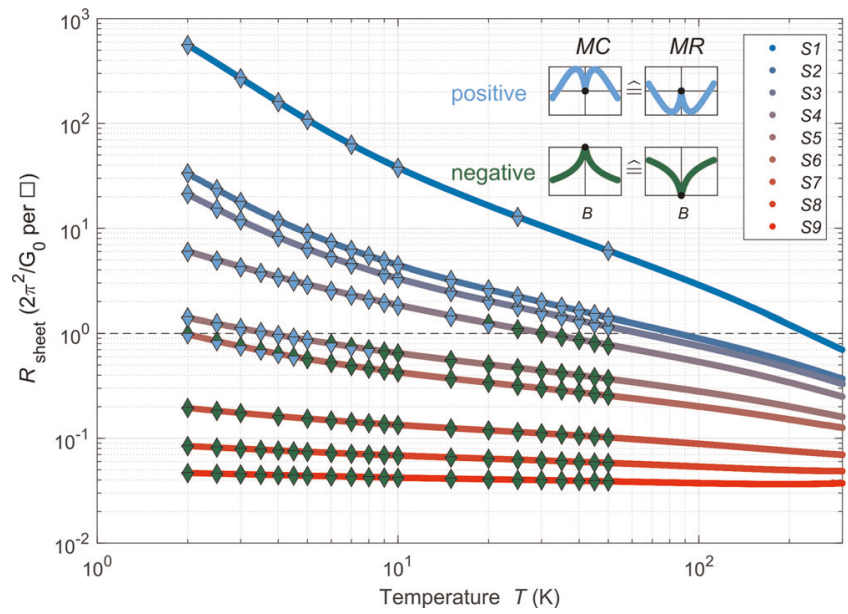

Fig. 2 Resistance versus temperature and the sign of the magnetoconductance with applied parallel and perpendicular fields for $\mathrm{SnSb}_{2} \mathrm{Te}_{4}$ samples. The degree of disorder determines the temperature dependence of the resistance as well as the sign of the magnetoconductance. With decreasing disorder, samples show a transition from hopping transport to diffusive transport (sample S1 to 59). This transition is accompanied by a sign change of the magnetoconductance (MC) from positive (blue) to negative (green), from the lowest temperatures up to $30 \mathrm{~K}$. The magnetic field direction is indicated with upward (downward) triangles for perpendicular (parallel) fields.

Magnetotransport in the diffusive regime

To understand the evolution of the sign and isotropy of the MC, we first examine the diffusive samples (S7-S9) whose 2D conductance is well above $0.5 G_{0} \pi^{-2}$. Here corrections to the conductance arising from WAL result in a magnetoconductance $\Delta G(B)$ given by: ${ }^{16,23}$

$$
\Delta G\left(B_{\perp}\right)=\frac{-a \cdot G_{0}}{2 \pi^{2}}\left[\psi\left(\frac{1}{2}+\frac{B_{\mathrm{el}}}{B_{\perp}}\right)-\frac{3}{2} \psi\left(\frac{1}{2}+\frac{\frac{4}{3} B_{\mathrm{so}}+B_{\Phi}}{B_{\perp}}\right)+\frac{1}{2} \psi\left(\frac{1}{2}+\frac{B_{\Phi}}{B_{\perp}}\right)\right]
$$

for perpendicular applied fields $B_{\perp}$. The characteristic fields $B_{\phi}, B_{\text {so }}$ and $B_{\mathrm{el}}$ correspond to dephasing, spin-orbit, and elastic scattering lengths through $B_{\mathrm{x}}=\hbar\left(4 \mathrm{e} \mathrm{l}_{\mathrm{x}}^{2}\right)^{-1}(\mathrm{x}=\phi$, so, el) and $a \approx 1$. For parallel field alignment WAL can be detected due to the finite thickness $(d)$ of the film with: $:^{23,31,32}$

$$
\Delta G\left(B_{\|}\right)=\frac{G_{0}}{2 \pi^{2}}\left[\frac{3}{2} \cdot \ln \left(1+\frac{B_{\|}^{2} e d^{2}}{12 \hbar\left(\frac{4}{3} B_{\text {so }}+B_{\phi}\right)}\right)-\frac{1}{2} \cdot \ln \left(1+\frac{B_{\|}^{2} e d^{2}}{12 \hbar B_{\phi}}\right)\right]
$$

Magnetoconductance data, as well as fits to Eqs. 1, 2, are plotted in Fig. 3a for sample $S 9$ as a function of temperature, and for samples $56-59$ at $2 \mathrm{~K}$ in Fig. 3b. (Additional fitting details and results are presented in the Supplementary Note 5.) The dashed lines are model (Eqs. 1, 2) fits that determine $B_{\text {so }}$ and $B_{\phi}$ and show excellent agreement with the measurements for both parallel and perpendicular fields. With increasing disorder (samples S6-S9, Fig. 3b) the cusp and saturation of the $M C$ vanish, revealing the disappearance of WAL approaching the resistance of $2 \pi^{2} / G_{0}$. Furthermore, the anisotropy of the $M C$ decreases with increasing disorder (Supplementary Fig. 12). Eventually, the signature of WAL disappears with increasing disorder, and (as shown in Fig. 2) the $\mathrm{MC}$ becomes positive as the perturbative analysis of diffusive behavior breaks down ${ }^{9}$ (Fig. 3b sample S6).

Magnetotransport in the hopping regime

While the negative $M C$ in the diffusive regime is easily ascribed to WAL, explaining the sign and characteristic shape of the positive
$M C$ in the hopping regime (i.e. samples with $G<<0.5 G_{0} \pi^{-2}$ ) is significantly more challenging. Figure $3 c$ shows the magnetoconductance of the most insulating sample $S 1$ which increases upon decreasing temperature between 25 and $2 \mathrm{~K}$. Decreasing the disorder for the samples in the hopping regime (samples S1-S5) results in a decreased magnitude of the positive $M C$ as seen in Fig. $3 \mathrm{~d}$, in striking contrast to the diffusive samples $57-59$ shown in Fig. $3 \mathrm{~b}$, where decreasing disorder causes an increased magnitude of the negative MC. In the insulating samples, the low-field magnetoconductance is positive everywhere and isotropic, showing that the low-field MC mechanism in this strongly disordered limit must be both small and isotropic.

Crossovers in the $\mathrm{MC}$ sign at distinct resistances have been observed in strongly interacting, ${ }^{30}$ topologically nontrivial materials with strong spin-orbit coupling, ${ }^{28}$ as well as in InSb quantum wells; ${ }^{29}$ doping $\ln _{2} \mathrm{O}_{3-x}$ with $\mathrm{Au}$ also produces a $\mathrm{MC}$ sign change, but one not associated with a change in transport mechanism. ${ }^{33}$ In all of these studies, including the present one, the negative MC in the diffusive regime has been attributed to WAL. Significant differences exist, however, regarding the strongly localized samples where a positive $\mathrm{MC}$ has been observed. Notably, previous studies report on an anisotropic MC, while an isotropic $M C$ is detected here.

The isotropy of the positive MC excludes orbital mechanisms, like path interference in the hopping regime, which are sensitive to the film thickness, ${ }^{34-37}$ as observed in the aforementioned studies, $28-30$ for disordered $\ln _{2} \mathrm{O}_{3}{ }^{33,38,39}$ or amorphous $\mathrm{NbSi}$ alloys. ${ }^{40}$ In the hopping regime path interference effects lead to an anisotropic MC scaling with the magnetic flux enclosed by the effective interference area, which is proportional to the product of hopping length and the square root of hopping length times localization length. ${ }^{34}$ Hence in thin films these effects are stronger for perpendicular fields. An isotropic MC would only be expected if the film thickness is larger than the hopping length. ${ }^{36,38,39}$ Given the small $(8.8 \mathrm{~nm})$ film thickness compared to hopping lengths of e.g. $\approx 17 \mathrm{~nm}$ for $S 1$ at $2 \mathrm{~K}$ (see also Supplementary Note 6) and the lack of a dominant perpendicular contribution, this mechanism can be excluded as the cause for the positive MC observed here (Fig. 3d sample S5). The weakness of the effect also eliminates other isotropic effects like magnetic dopants ${ }^{41}$ or the Zeeman effect as possible causes; the latter has been associated with disordered topological insulators. ${ }^{42}$

\section{Spin memory effect}

Isotropic magnetotransport might also be related to the spin alignment in a magnetic field. Indeed in highly disordered systems a spin-sensitive hopping mechanism has recently been proposed. ${ }^{43}$ This effect relies on the variation of the gyromagnetic factor $\delta g$, caused by pronounced disorder and neglects electron interaction effects. ${ }^{43}$ PCMs such as $\mathrm{SnSb}_{2} \mathrm{Te}_{4}$ are characterized by large levels of structural disorder and a high static dielectric constant, e.g. 47 even in amorphous $\mathrm{GeSb}_{2} \mathrm{Te}_{4}{ }^{44}$ and expected to be higher in the crystalline phase, ${ }^{17,45,46}$ which minimizes electron correlation effects between adjacent sites. Hence, such samples present a unique opportunity to resolve effects that require inhomogeneity in the local spin environment.

In VRH transport, electrons tunnel from an occupied to an empty site, with the energy provided by an absorbed phonon. ${ }^{9}$ In this process, the hopping distance is temperature dependent, as for lower temperatures the initial and final state energies have to be closer together, due to the smaller average phonon energy available. ${ }^{9}$ This causes longer hopping distances at lower temperature (Fig. 4a). In spin-correlated hopping (SCH) the hopping probability between site $i$ and $j$ also depends on the spin on a nearby site $\mathrm{ij}$, called a link spin. If a virtual singlet state of the electrons at site $\mathrm{i}$ and ij can be formed, the total transition rate from site $\mathrm{i}$ to $\mathrm{j}$ is increased compared to the virtual triplet 
a

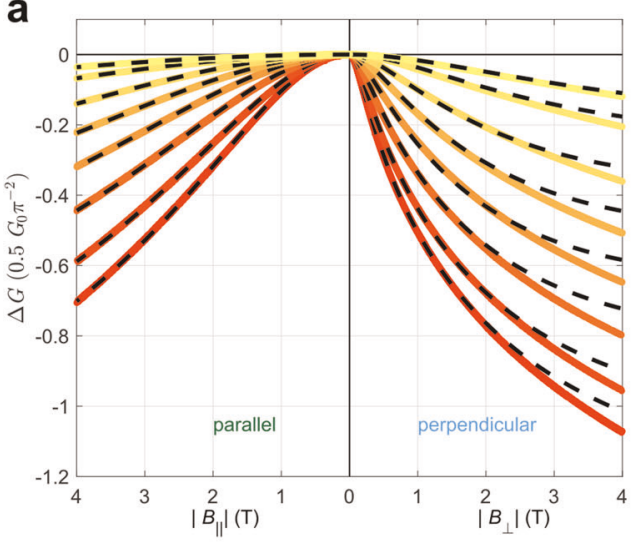

C

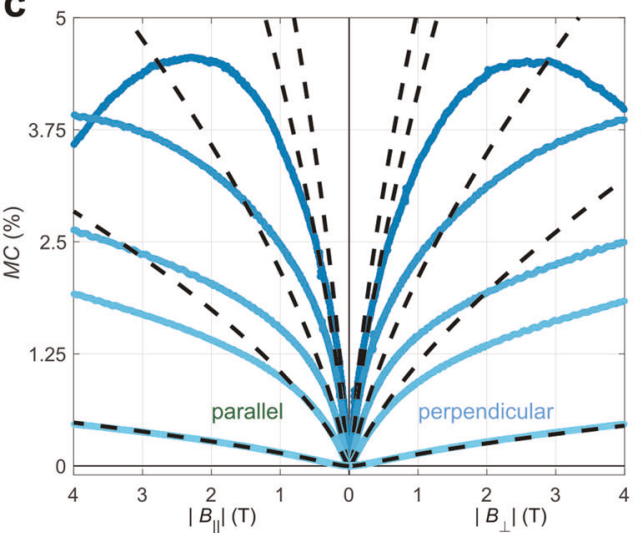

b

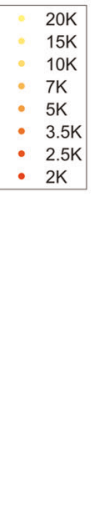

$\begin{array}{r}-2 \mathrm{~K} \\ : 3 \mathrm{~K} \\ : 4 \mathrm{~K} \\ -7 \mathrm{~K} \\ \hline\end{array}$

d
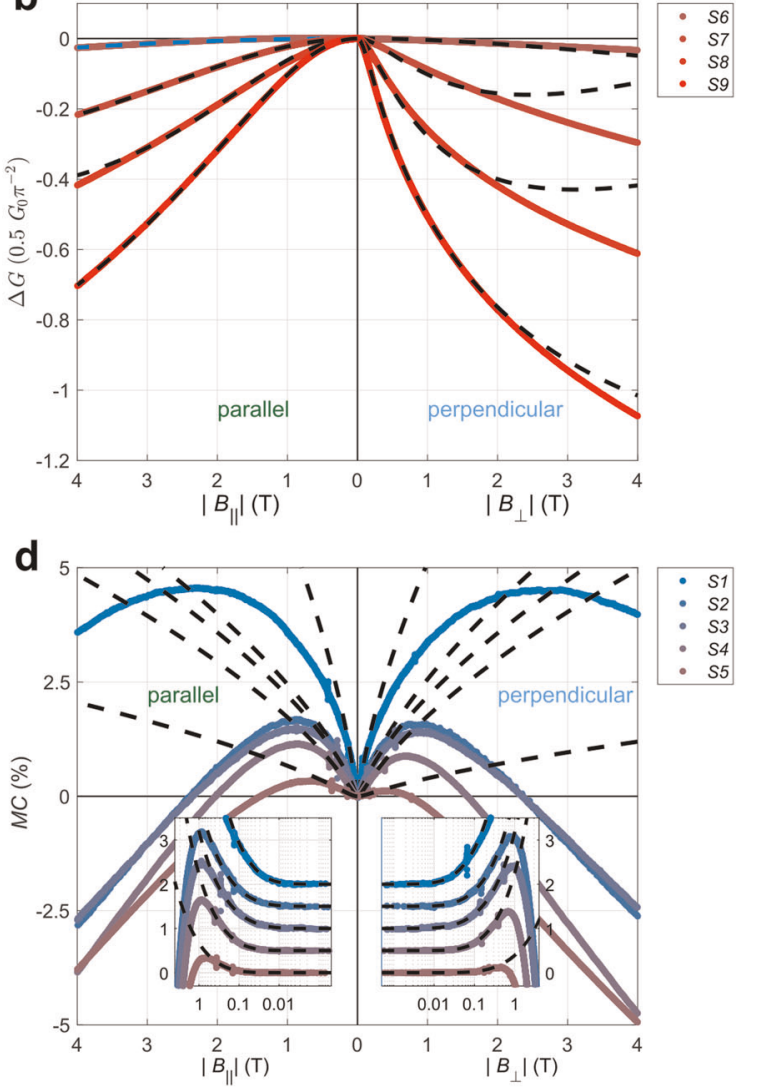

Fig. 3 Magnetoconductance of diffusive and insulating $\mathrm{SnSb}_{2} \mathrm{Te}_{4}$ samples. Solid curves are measured data, while dashed lines represent model fits. a The temperature-dependent MC for the most metallic sample $S 9$ is dominated by weak antilocalization. $\mathbf{b}$ With increasing disorder, the $2 \mathrm{~K}$ magnetoconductance for samples $S 6-S 9$ shows a steady decline in the contribution of WAL, as well as a decrease in anisotropy. The blue dashed line for sample $S 6$ indicates the change to positive MC at lowest fields (see Supplementary Note 4). c The most insulating sample 51 shows a low-field MC that is positive and isotropic. The fits (dashed lines) to Eq. 4 at lowest fields show excellent agreement with the data (apart from a negative slope that appears in fields above $2 \mathrm{~T}$ arising from wave function shrinkage). $\mathbf{d}$ Strongly and marginally insulating $\mathrm{SnSb}_{2} \mathrm{Te}_{4}$ samples at $2 \mathrm{~K}$. The magnitude of the MC increases while the anisotropy of the MC decreases with increasing disorder. The insets in $\log (B)$-scale demonstrate the excellent agreement of theory and data at lowest fields; inset curves are shifted for clarity.

configuration (Fig. 4b). These spin correlations between site $i$ and ij will persist, as long as the spin relaxation rate $\left(1 / \tau_{\mathrm{s}}\right)$ is much slower than the hopping rate $\left(1 / \tau^{* *}\right)$ between the states, i.e. if $\tau^{* *}<<\tau_{s}$. Hence, electrons will remain in the unfavorable triplet spin configuration until $\tau_{\mathrm{s}}$, thus hopping for virtual triplet states is dominated by direct transitions from i to $\mathrm{j}$.

The application of a magnetic field suppresses these spin correlation effects through the variation of the local fields caused by the spatial variation in $\delta g$ Fig. 4c. The electron's spin will decohere on a timescale shorter than $\tau_{s}$, resulting in a possible change of the spin configuration from a triplet to a singlet state, increasing the conductance. The spin sensitivity of the effect renders it insensitive to the magnetic field orientation relative to the sample, consistent with the data in Fig. 3c, d. In SST, the variation of the gyromagnetic factor $\delta g$ can be attributed to the presence of randomly distributed vacancies, that vary the influence of the magnetic field on the tunneling electron. In the simplest picture of this effect, spin correlations are suppressed in a magnetic field, with a MC predicted to be: ${ }^{43}$

$$
\frac{\delta G(B)}{G(0)} \approx 3.9 \cdot A \cdot\left[\left(\frac{\mathrm{i} B}{B^{* *}}+\frac{B_{\mathrm{s}}}{B^{* *}}\right)^{0.65}+\left(-\frac{\mathrm{i} B}{B^{* *}}+\frac{B_{\mathrm{s}}}{B^{* *}}\right)^{0.65}-2 \cdot\left(\frac{B_{\mathrm{s}}}{B^{* *}}\right)^{0.65}\right]
$$

where $B^{* *}$ is associated with the hopping time to the unoccupied state $\tau^{* *}$ via $B^{* *}=\hbar\left(\delta g \mu_{\mathrm{B}} \tau^{* *}\right)^{-1}$, and $B_{\mathrm{s}}$ is determined by the spinlifetime $\tau_{\mathrm{s}}$ via $B_{S}=\hbar\left(\delta g \mu_{\mathrm{B}} \tau_{\mathrm{s}}\right)^{-1}$. The magnitude of the effect (captured in the prefactor $A \sim \gamma^{2} p$ ) depends on the probability $p$ of having a link spin and the corresponding hopping amplitude $\gamma^{2}$, both considered to be small in the derivation of Eq. 3. It can be assumed that for decreasing temperatures $p$ increases as more states become localized that could act as link spins. The lifetimes $\tau^{* *}(T)$ and $\tau_{\mathrm{s}}(T)$ are expected to increase with decreasing temperature, ${ }^{9,43}$ which would result in a decrease of $B^{* *}$ and $B_{\mathrm{s}}$.

In order to reduce the fitting parameters, we introduce a generalized hopping parameter $M=3.9 A\left(B^{* *}\right)^{-0.65}$, which results in:

$$
\frac{\delta G(B)}{G(0)} \approx M \cdot\left[\left(\mathrm{i} B+B_{\mathrm{s}}\right)^{0.65}+\left(-\mathrm{i} B+B_{\mathrm{s}}\right)^{0.65}-2 \cdot\left(B_{\mathrm{s}}\right)^{0.65}\right]
$$

This model shows excellent quantitative agreement with the experimental data in Fig. 3c, d over a broad range of magnetic fields (see Supplementary Note 7 for fitting details). This is strong support for the existence of spin memory hopping, as evidenced by the destruction of spin correlations.

\section{DISCUSSION}

Furthermore, we can analyze the dependence of the fitting parameters of Eq. $4\left(B_{\mathrm{s}}\right.$ and $M$ ) on temperature and disorder and compare it to the corresponding dependence of the inelastic scattering field $B_{\phi}$ on the diffusive side on these parameters. From this analysis we also obtain the dependence of the underlying physical quantities in the hopping regime, i.e. $\tau_{s}, \tau^{* *}$ and $\delta g$ on temperature, and annealing temperature respectively. The 

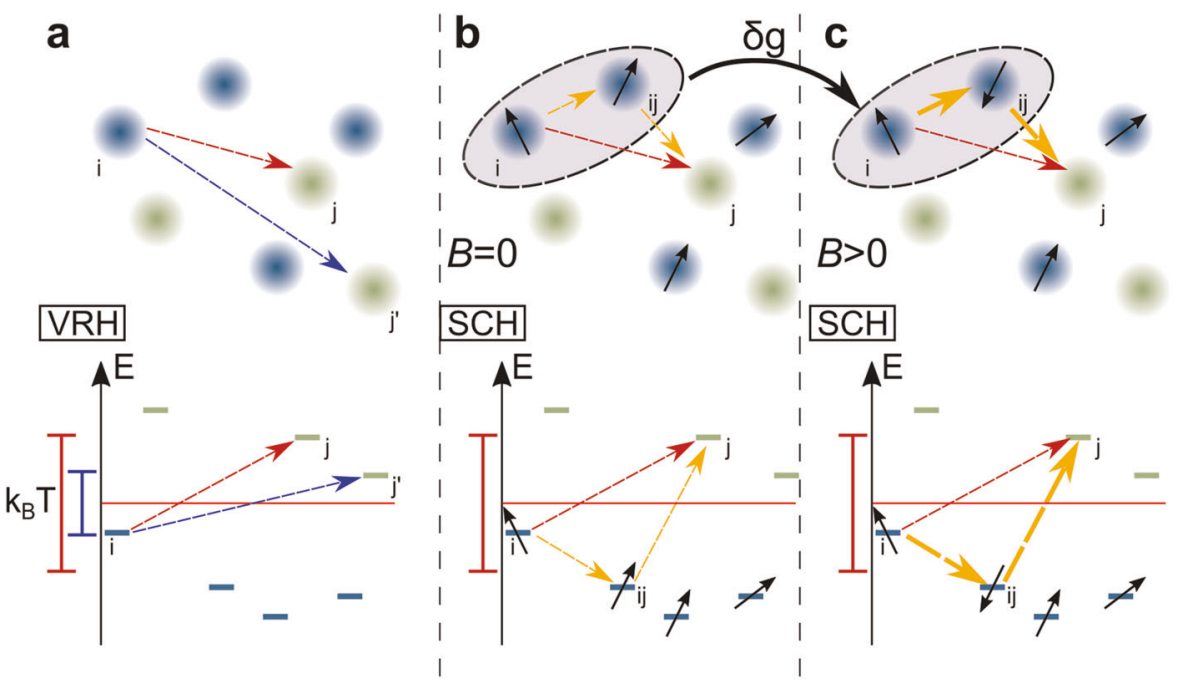

Fig. 4 Sketch of hopping transport mechanisms in real (top) and energy space (bottom). a In variable range hopping an electron tunnels from one occupied site (blue) to an unoccupied site (green). Reducing the temperature decreases the number of energetically accessible states when hopping, which leads to a larger hopping length. b For spin-memory assisted hopping, the spin configuration with an adjacent occupied site becomes relevant as the total transition rate is influenced by the virtual state of $\mathrm{i}$ and ij (ellipsis). In particular, the probability of an indirect transition from $\mathrm{i}$ to $\mathrm{j}$ via ij (orange path) is significantly reduced, if $\mathrm{i}$ and $\mathrm{ij}$ form a virtual triplet state. Spin correlations hinder the alteration of the spin state over time. c The application of a magnetic field in the presence of a spatially varying g-factor ( $\delta g$ ) destroys these spin correlations e.g. the spin orientation at site ij changes and increases the total transition rate from site i to $j$, if a virtual singlet state of $i$ and ij can be formed.

prefactor $M$ and characteristic field $B_{\mathrm{s}}$ extracted from this model exhibit a temperature and disorder evolution that is consistent with their physical origin. The effective hopping factor $M$ increases with decreasing temperature, as shown in Fig. 5a. The decrease in temperature, as seen in Fig. $5 \mathrm{~b}$ results in a decrease of $B_{s}$, which resembles the dephasing field in the diffusive regime $B_{\phi}$ that also decreases (Fig. 5c). The expected evolution of electron-electron $(T)$ and electron-phonon $\left(\sim T^{2}\right)$ dominated dephasing in $B_{\phi}$ (dashed lines) are also shown in Fig. $5 c$ (for further details see Supplementary Note 5). The temperature dependence of the quantities of Eq. 4 can be attributed to changes of the relevant time scales, as the reduction of $B_{\mathrm{s}}$ and the upturn of $M$ are both consistent with the predicted increase of $\tau^{* *}(T)$ and $\tau_{\mathrm{s}}(T)$.

The dependence of $M, B_{\mathrm{s}}$ and $B_{\phi}$ on disorder illustrates the difference between the diffusive and strongly localized pictures. While $B_{\phi}$ on the diffusive side decreases with decreasing disorder, $B_{\mathrm{s}}$ on the strongly localized side decreases with increasing disorder. A decrease of $B_{\mathrm{s}}$ with increasing disorder should result from an increase of the spatial variation of the gyromagnetic-factor $\delta g$ through e.g. larger displacement of vacancies, which can also contribute to an increase of $M$. Looking at the magnitude of the values of $M<<1 \mathrm{~T}^{-0.65}$ and $B_{\mathrm{s}}<<1 \mathrm{~T}$ in Fig. $5 \mathrm{a}, \mathrm{b}$, we can assume that both the prefactor $A<1$ being small and $B_{\mathrm{s}}<B^{* *}$ should be fulfilled, the latter being equivalent to $\tau^{* *}<\tau_{\mathrm{s}}$, thus verifying the existence of a well-defined spin-lifetime.

The spin lifetime being longer than the hopping time in conjunction with the disordered character of the localized states, could be interpreted as a glassy spin phenomenon, which should also be experimentally traceable in e.g. relaxation time experiments, ${ }^{7}$ but wasn't observable in a hysteresis of the MC in this study. The relevance of the local spin environment on hopping should also be important in similarly strongly disordered systems. Finally, we note that there is an anisotropic MC component at elevated fields above 1T that decreases in magnitude as the samples become more disordered (Fig. 3d). This is consistent with wave function shrinkage with applied field, which reduces the overlap of the wave functions transverse to the field direction. ${ }^{9,40}$ For $S 5$ at $2 \mathrm{~K}$ and $4 \mathrm{~T}$ the effect of wave function shrinkage is estimated to be $1.6 \%$ (for further considerations see
Supplementary Note 8). A complete model of both the shrinkage and spin-memory effects may thus be sufficient to describe the behavior observed over a wide range of magnetic fields.

In summary, the insulating crystalline phase change material SST exhibits an isotropic positive MC which increases with disorder, in striking contrast to the diffusive transport regime of the same material. In metallic samples, WAL leads to an anisotropic and negative MC, which decreases with increasing disorder. The isotropic positive MC in the hopping regime, on the contrary, is attributed to destruction of spin correlations. ${ }^{43}$ This finding provides new insights on the transport of spin and charge in strongly disordered insulators and presents striking experimental evidence for the significance of a spin memory lifetime. Apparently, spin correlations influence the transport on the insulating side, when large amounts of disorder and small electronic correlations prevail. In crystalline $\mathrm{SnSb}_{2} \mathrm{Te}_{4}$, these conditions are met due to the high number of disordered vacancies, in conjunction with a bonding mechanism which ensures very low electronic correlations. ${ }^{45,46}$ Thus, upon decreasing disorder, a transition from hopping to diffusive transport is observed, one which is accompanied by an MC sign change and a change of MC isotropy. We expect similar phenomena whenever electronic correlations are small, e.g. the static dielectric constant is large, effectively screening charge carriers, and the possibility for pronounced structural disorder exists. Such studies hence promise further insights on the nature of the strong localization transition in electronic systems and can unravel the nature of the glassy spin dynamics of localized states.

\section{METHODS}

- Samples were grown using $\mathrm{DC}$ magnetron sputtering of $\mathrm{SnSb}_{2} \mathrm{Te}_{4}$, from a stoichiometric target on Si-100 wafers with a $1 \mu$ m thick $\mathrm{SiO}_{2}$ layer for electrical insulation. In-situ capping used $33 \mathrm{~nm}$ (S1-S3) and $15 \mathrm{~nm}$ (S4-S9) thick layers of $\mathrm{ZnS}: \mathrm{SiO}_{2}$ 80:20. The films were crystalline as deposited and post-annealed in a tube furnace under flowing $\mathrm{Ar}$ atmosphere (0.1-0.2 l/ min) with a heating rate of $2 \mathrm{~K} / \mathrm{min}$ and a holding time of $3 \mathrm{~h}$. The sample thickness was determined with $X$-ray reflectometry measurements on a Philips X'Pert Analytical x-ray setup. The thickness of both sputter runs 
a

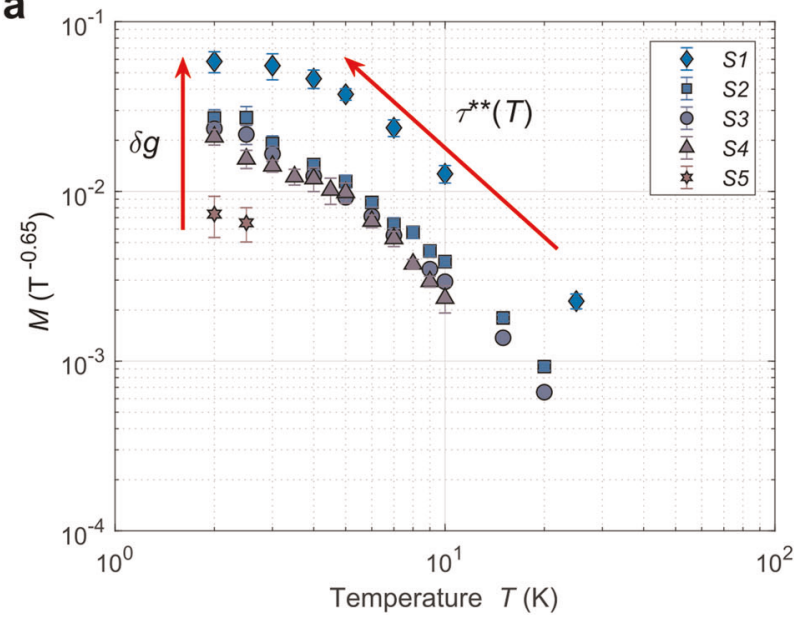

b

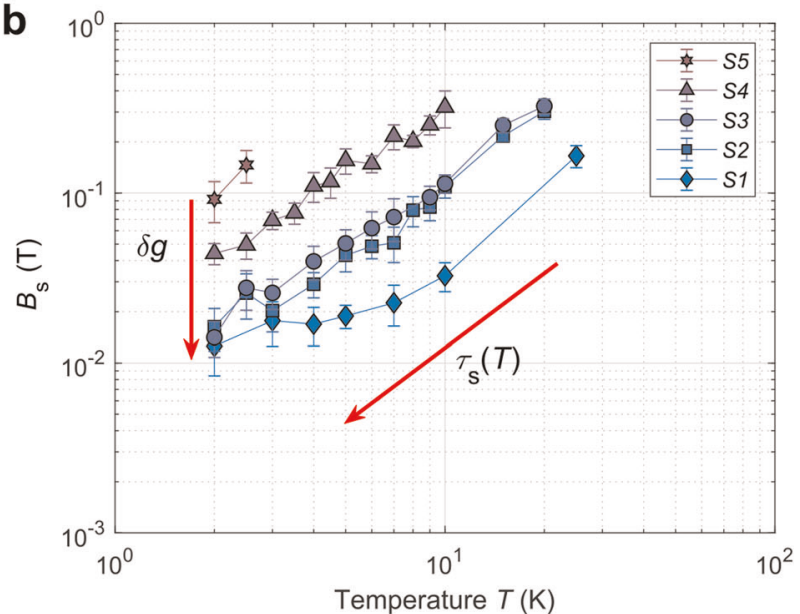

C

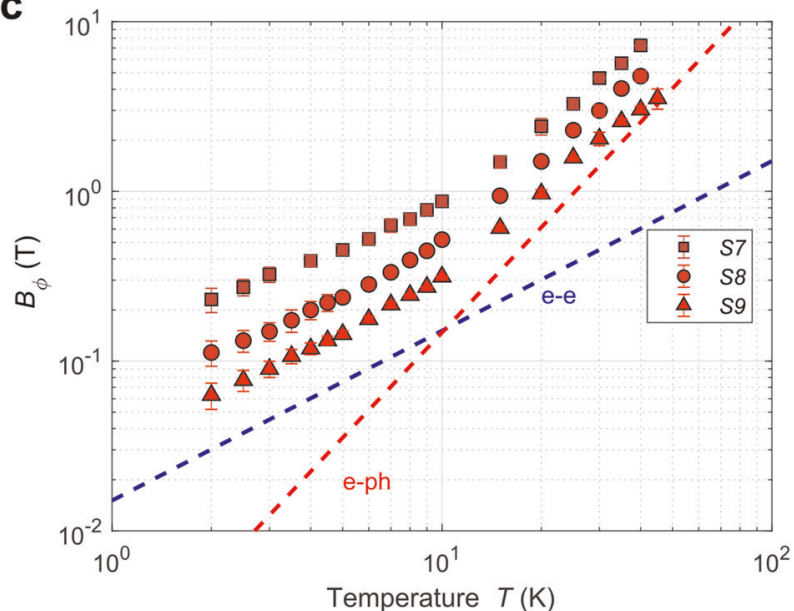

was determined to be $8.8 \pm 0.5 \mathrm{~nm}$, limited by the analytical method. The rock-salt crystal structure was confirmed with X-ray diffraction under grazing incidence (same instrument). Apart from an expected slight decrease of the lattice constant with increasing annealing temperature, no structural transition could be observed.

- Hall bar devices were fabricated with a combination of photolithography, Ar ion milling and chemical etching with hydrofluoric acid. Ohmic contacts were patterned on top of the devices. The contacts consist of a $\mathrm{Cr}$-adhesion bottom layer and a thicker Au top layer. We measured a total of 17 samples; data shown in this work are representative.
Fig. 5 Temperature dependence of characteristic dephasing and spin fields. a Temperature evolution of the prefactor $M$ from Eq. 4; data are only shown for samples that clearly exhibit the spinmemory effect and are the average of perpendicular and parallel component. For the least insulating sample $S 5$, this corresponds to only the lowest temperatures measured $(2-3 \mathrm{~K})$. The arrows indicate the influence of increasing disorder (vertical) associated with an increase of $\delta g$, or decreasing temperature (oblique) leading to increasing timescales, on the parameters. $\mathbf{b}$ The characteristic spinhopping fields decrease with decreasing temperature; for the same measurements as in a. c Dephasing fields for the diffusive samples S7-S9 taken as average of perpendicular and parallel contributions. Model dependences are shown as dashed lines that arise from electron-electron (blue) and electron-phonon (red) dephasing. Error bars represent estimates of the $\pm 1 \sigma$ uncertainty of the parameters from fits.

- Transport measurements were performed in a Dynacool ${ }^{\mathrm{TM}}$ from Quantum Design $^{\circledR}$, with the electrical-transport-option (ETO) extension at low frequencies $0.4-0.5 \mathrm{~Hz}$ and very low excitation currents of 10-100 nA. The sample orientation relative to the magnetic field was controlled with a temperature calibrated rotator insert, with an angular accuracy of $\sim 1^{\circ}$.

\section{DATA AVAILABILITY}

The data that support the findings of this study are available from the corresponding author upon reasonable request.

Received: 28 June 2019; Accepted: 3 October 2019; Published online: 02 December 2019

\section{REFERENCES}

1. Anderson, P. W. Absence of diffusion in certain random lattices. Phys. Rev. 109, 1492-1505 (1958).

2. Smith, J. et al. Many-body localization in a quantum simulator with programmable random disorder. Nat. Phys. 12, 907-911 (2016).

3. Semeghini, G. et al. Measurement of the mobility edge for 3D Anderson localization. Nat. Phys. 11, 554-559 (2015).

4. Jendrzejewski, F. et al. Three-dimensional localization of ultracold atoms in an optical disordered potential. Nat. Phys. 8, 398-403 (2012).

5. Hu, H., Strybulevych, A., Page, J. H., Skipetrov, S. E. \& van Tiggelen, B. A. Localization of ultrasound in a three-dimensional elastic network. Nat. Phys. 4, 945-948 (2008).

6. Sperling, T., Bührer, W., Aegerter, C. M. \& Maret, G. Direct determination of the transition to localization of light in three dimensions. Nat. Photon. 7, 48-52 (2013).

7. Dobrosavljevic, V., Trivedi, N. \& Valles Jr., J. M. Conductor Insulator Quantum Phase Transitions. (Oxford University Press, 2012).

8. Mott, N. Metal-insulator Transitions. (Taylor \& Francis, 1990).

9. Shklovskii, B. I. \& Efros, A. L. Electronic Properties Of Doped Semiconductors. (Springer-Verlag Berlin, 1984).

10. Punnoose, A. \& Finkel'stein, A. M. Metal-insulator transition in disordered twodimensional electron systems. Science 310, 289-291 (2005).

11. Spivak, B., Kravchenko, S. V., Kivelson, S. A. \& Gao, X. P. A. Colloquium: transport in strongly correlated two dimensional electron fluids. Rev. Mod. Phys. 82, 1743-1766 (2010).

12. Melnikov, M. Y. et al. Quantum phase transition in ultrahigh mobility SiGe/Si/SiGe two-dimensional electron system. Phys. Rev. B 99, 081106 (2019).

13. Bergmann, G. Weak localization in thin films: a time-of-flight experiment with conduction electrons. Phys. Rep. 107, 1-58 (1984).

14. Lee, P. A. \& Ramakrishnan, T. V. Disordered electronic systems. Rev. Mod. Phys. 57, 287-337 (1985).

15. Gantmakher, V. F. Electrons and Disorder in Solids. (Oxford University Press, 2005).

16. Hikami, S., Larkin, A. I. \& Nagaoka, Y. Spin-orbit interaction and magnetoresistance in the two dimensional random system. Prog. Theor. Phys. 63, 707-710 (1980).

17. Siegrist, T. et al. Disorder-induced localization in crystalline phase-change materials. Nat. Mater. 10, 202-208 (2011). 
18. Zhang, W. et al. Role of vacancies in metal-insulator transitions of crystalline phase-change materials. Nat. Mater. 11, 952-956 (2012).

19. Volker, H., Jost, P. \& Wuttig, M. Low-temperature transport in crystalline $\mathrm{Ge}_{1} \mathrm{Sb}_{2} \mathrm{Te}_{4}$. Adv. Funct. Mater. 25, 6390-6398 (2015).

20. Jost, P. et al. Disorder-induced localization in crystalline pseudo-binary GeTe-Sb $\mathrm{Te}_{3}$ alloys between $\mathrm{Ge}_{3} \mathrm{Sb}_{2} \mathrm{Te}_{6}$ and GeTe. Adv. Funct. Mater. 25, 6399-6406 (2015).

21. Dück, M. M. et al. Disorder control in crystalline $\mathrm{GeSb}_{2} \mathrm{Te}_{4}$ and its impact on characteristic length scales. Phys. Status Solidi RRL-Rapid Res. Lett. 13, 1800578 (2019). https://doi.org/10.1002/pssr.201800578.

22. Bragaglia, V. et al. Metal - insulator transition driven by vacancy ordering in GeSbTe phase change materials. Sci. Rep. 6, 23843 (2016).

23. Breznay, N. P. et al. Weak antilocalization and disorder-enhanced electron interactions in annealed films of the phase-change compound $\mathrm{GeSb}_{2} \mathrm{Te}_{4}$. Phys. Rev. B 86, 205302 (2012)

24. Wuttig, M. \& Yamada, N. Phase-change materials for rewriteable data storage. Nat. Mater. 6, 824-832 (2007).

25. Burr, G. W. et al. Phase change memory technology. J. Vac. Sci. Technol. B 28, 223-262 (2010)

26. Hornung, M. \& Löhneysen, H. Crossover from Mott to Efros-Shklovskii variable range-hopping in Si:P. Czechoslovak. J. Phys. 46, 2437-2438 (1996).

27. Zhang, Y., Dai, O., Levy, M. \& Sarachik, M. P. Probing the Coulomb gap in insulating n-type CdSe. Phys. Rev. Lett. 64, 2687-2690 (1990).

28. Liao, J. et al. Observation of Anderson localization in ultrathin films of threedimensional topological insulators. Phys. Rev. Lett. 114, 216601 (2015).

29. Ishida, S., Manago, T., Nishizako, N., Geka, H. \& Shibasaki, I. Spin-orbit interaction and negative magnetoresistance for localized electrons in InSb quantum wells. Phys. E Low.-Dimens. Syst. Nanostruct. 42, 984-986 (2010).

30. Hsu, S.-Y. \& Valles, J. Observation of a well defined transition from weak to strong localization in two dimensions. Phys. Rev. Lett. 74, 2331-2334 (1995).

31. Gershenzon, E. M., Gubankov, V. N. \& Zhuravlev, Y. U. E. Effects of 'weak' localization and electron-electron Interaction in thin copper and silver films. Sov. Phys. JETP 56, 1362-1369 (1982).

32. Rosenbaum, R. Superconducting fluctuations and magnetoconductance measurements of thin films in parallel magnetic fields. Phys. Rev. B 32, 2190-2199 (1985).

33. Shapir, Y. \& Ovadyahu, Z. Effects of spin-orbit scattering on hopping magnetoconductivity. Phys. Rev. B 40, 12441-12445 (1989).

34. Nguen, V. L., Spivak, B. Z. \& Shklovskii, B. I. Tunnel hopping in disordered systems. Sov. Phys. JETP 62, 1021-1029 (1985).

35. Nguyen, V. L., Spivak, B. Z. \& Shklovskii, B. I. Aaronov-Bohm oscillations with normal and superconducting flux quanta in hopping conductivity. JETP Lett. 41, 42-45 (1985)

36. Schirmacher, W. Quantum-interference magnetoconductivity in the variablerange-hopping regime. Phys. Rev. B 41, 2461-2468 (1990).

37. Meir, Y., Wingreen, N. S., Entin-Wohlman, O. \& Altshuler, B. L. Spin-orbit scattering for localized electrons: absence of negative magnetoconductance. Phys. Rev. Lett. 66, 1517-1520 (1991).

38. Vaknin, A., Frydman, A., Ovadyahu, Z. \& Pollak, M. High-field magnetoconductance in Anderson insulators. Phys. Rev. B 54, 13604-13610 (1996).

39. Ovadyahu, Z. Quantum coherent effects in Anderson insulators. Waves Random Media 9, 241-253 (1999).

40. Pounder, N. M. \& Howson, M. A. Negative magnetoresistance in NbSi amorphous alloys. J. Phys. Condens. Matter 3, 2069-2073 (1991).

41. Oiwa, A. et al. Nonmetal-metal-nonmetal transition and large negative magnetoresistance in (Ga, Mn) As/GaAs. Solid State Commun. 103, 209-213 (1997)

42. Breunig, O. et al. Gigantic negative magnetoresistance in the bulk of a disordered topological insulator. Nat. Commun. 8, 15545 (2017).
43. Agam, O., Aleiner, I. L. \& Spivak, B. Spin-memory effect and negative magnetoresistance in hopping conductivity. Phys. Rev. B 89, 100201 (2014).

44. Chen, $C$. et al. Dielectric properties of amorphous phase-change materials. Phys. Rev. B 95, 094111 (2017)

45. Wuttig, M., Deringer, V. L., Gonze, X., Bichara, C. \& Raty, J.-Y. Incipient metals: functional materials with a unique bonding mechanism. Adv. Mater. 30, 1803777 (2018).

46. Raty, J.-Y. et al. A quantum-mechanical map for bonding and properties in solids. Adv. Mater. 31, 1806280 (2019).

\section{ACKNOWLEDGEMENTS}

We thank Riccardo Mazzarello and Boris Spivak for insightful scientific discussions, and T. D. Donnelly for critical feedback on the manuscript. The research leading to these results has received funding from the European Union Seventh Framework Programme (FP7/2007-2013) under grant agreement no. 340698 as well as the Deutsche Forschungsgemeinschaft through SFB 917 ('Nanoswitches').

\section{AUTHOR CONTRIBUTIONS}

The project plan was designed by J.R., H.V. and M.W. The samples where processed, characterized and measured by J.R. The data was evaluated by J.R., with the assistance of H.V. and N.P.B. The manuscript was written by J.R., N.P.B. and M.W.; all authors contributed to the discussion of the results for the manuscript.

\section{COMPETING INTERESTS}

The authors declare no competing interests.

\section{ADDITIONAL INFORMATION}

Supplementary information is available for this paper at https://doi.org/10.1038/ s41535-019-0196-6.

Correspondence and requests for materials should be addressed to M.W.

Reprints and permission information is available at http://www.nature.com/ reprints

Publisher's note Springer Nature remains neutral with regard to jurisdictional claims in published maps and institutional affiliations.

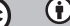

Open Access This article is licensed under a Creative Commons Attribution 4.0 International License, which permits use, sharing, adaptation, distribution and reproduction in any medium or format, as long as you give appropriate credit to the original author(s) and the source, provide a link to the Creative Commons license, and indicate if changes were made. The images or other third party material in this article are included in the article's Creative Commons license, unless indicated otherwise in a credit line to the material. If material is not included in the article's Creative Commons license and your intended use is not permitted by statutory regulation or exceeds the permitted use, you will need to obtain permission directly from the copyright holder. To view a copy of this license, visit http://creativecommons. org/licenses/by/4.0/.

(c) The Author(s) 2019 